\title{
Combining In Vivo Corneal Confocal Microscopy with Deep Learning-based Analysis Reveals Sensory Nerve Fiber Loss in Acute SIV Infection
}

Megan E. McCarron ${ }^{1 *}$, Rachel L. Weinberg ${ }^{1^{*}}$, Jessica M. Izzi ${ }^{1}$, Suzanne E. Queen ${ }^{1}$, Stuti L. Misra $^{2}$, Daniel B. Russakoff ${ }^{3}$, Jonathan D. Oakley ${ }^{3}$, Joseph L. Mankowski ${ }^{1}$

${ }^{*}$ Contributed equally

${ }^{1}$ Department of Molecular and Comparative Pathobiology, Johns Hopkins University School of Medicine, Baltimore, MD

${ }^{2}$ Department of Ophthalmology, Faculty of Medical and Health Sciences, New Zealand National Eye Centre, University of Auckland, Auckland, New Zealand

${ }^{3}$ Voxeleron LLC, San Francisco, CA

Correspondence: Joseph L Mankowski

Department of Molecular and Comparative Pathobiology, Johns Hopkins University, Baltimore, MD

Tel.: 4109559770

Fax: 4109559823

e-mail: imankows@jhmi.edu

Running title: Corneal confocal microscopy and deep-learning reveal nerve loss in SIV 
Key words: cornea, subbasal nerve fibers, confocal microscopy, deep learning, macaques, SIV

Grant Support. NIH R01NS097221, R01NS113703, U42OD013117, and a Blaustein Research Grant, Johns Hopkins University School of Medicine. Fulbright New Zealand provided support for Dr. Stuti Misra.

Acknowledgements. We thank Dr. Robert J. Adams, the JHU Retrovirus Lab team, and JHU Research Animal Resource members for their generous assistance in completing this project. 


\begin{abstract}
Purpose

To characterize corneal subbasal nerve plexus morphologic features using in vivo corneal confocal microscopy (IVCM) in normal and SIV-infected macaques and to implement automated assessments using novel deep learning-based methods customized for macaque studies.
\end{abstract}

\title{
Methods
}

In vivo corneal confocal microscopy images were collected from both male and female agematched specific-pathogen free rhesus and pigtailed macaques housed at the Johns Hopkins University breeding colony using the Heidelberg HRTIII with Rostock Corneal Module. We also obtained repeat IVCM images of 12 SIV-infected animals including pre-infection and 10 day post-SIV infection time-points. All IVCM images were analyzed using a novel deep convolutional neural network architecture developed specifically for macaque studies.

\section{Results}

Deep learning-based segmentation of subbasal nerves in IVCM images from macaques demonstrated that corneal nerve fiber length (CNFL) and fractal dimension measurements did not differ between species, but pigtailed macaques had significantly higher baseline corneal nerve fiber tortuosity than rhesus macaques $(P=0.005)$. Neither sex nor age of macaques was associated with differences in any of the assessed corneal subbasal nerve parameters. In the SIV/macaque model of HIV, acute SIV infection induced significant 
decreases in both corneal nerve fiber length and fractal dimension $(P=0.01$ and $P=0.008$ respectively).

\section{Conclusions}

The combination of IVCM and objective, robust, and rapid deep-learning analysis serves as a powerful noninvasive research and clinical tool to track sensory nerve damage, enabling early detection of neuropathy. Adapting the deep-learning analyses to human corneal nerve assessments will refine our ability to predict and monitor damage to small sensory nerve fibers in a number of clinical settings including HIV, multiple sclerosis, Parkinson's disease, diabetes, and chemotherapeutic neurotoxicity. 


\section{Introduction}

Human immunodeficiency virus (HIV) associated sensory neuropathy remains a common and debilitating complication of HIV infection, even with antiretroviral therapy (ART).(1, 2) Signs of HIV neuropathy can develop early during primary HIV infection before most patients start antiretroviral therapy.(3) Studies in SIV/macaque models of HIV PNS damage have demonstrated that SIV infects and replicates within macrophages in sensory ganglia during the first week of infection followed shortly thereafter by immune activation of macrophages, at day 10 post-infection.(3-6) Concordant loss of epidermal nerve fibers in the skin of the hind feet can be detected at 14 days post-SIV infection.(7)

The current gold standard to measure sensory nerve fiber loss in both research and clinical settings is to calculate epidermal nerve fiber density in skin biopsies after PGP9.5 immunostaining to identify the small sensory nerve fibers.(8) We have adapted and validated similar approaches for use in macaques; however, serial biopsies are problematic for animals and human subjects, as it requires repeat sampling within the same local region of sensory innervation. Accordingly, we have developed an alternative non-invasive approach to measure sensory fiber loss amenable to longitudinal tracking of individual animals using in vivo corneal confocal microscopy (IVCM). This technique has previously been utilized in human studies as a tool to detect early changes in corneal subbasal nerves prior to diabetic peripheral neuropathy and has great potential for tracking sensory fiber loss longitudinally in HIV patients.(3, 9-13). Therefore, IVCM can be used as a marker to potentially diagnose and monitor progression of peripheral neuropathy. Our prior work using $\beta$ III tubulin immunostained corneal whole mount sections coupled with automated analysis first 
demonstrated that alterations in nerve fiber density of the subbasal plexus of the cornea correlated with epidermal nerve fiber loss in SIV animals that progressed to AIDS.(14) This work extends these methods and approach to in vivo imaging. The aims of the current study were to establish reference normal values for macaques and to investigate potential SIVinduced changes in the corneal subbasal nerve plexus using IVCM combined with novel deep learning-based methods customized for image processing for macaque studies.

\section{Materials and Methods}

Animals. All uninfected macaques used in this study were housed in indoor-outdoor enclosures in harem breeding groups at the Johns Hopkins University (JHU) Research Farm. Pigtailed macaques (Macaca nemestrina) and rhesus macaques (Macaca mulatta) were housed separately. Animals were screened annually and consistently tested serologically negative for Macacine herpesvirus 1 (B virus), simian immunodeficiency virus (SIV), simian T-cell leukemia virus (STLV-1), and simian retrovirus (SRV), comprising the battery of pathogens excluded to establish specific-pathogen free (SPF) status. Intradermal tuberculin skin tests were performed semi-annually and all animals in the colony were consistently negative.

Animal study groups: IVCM images were obtained from 21 healthy rhesus macaques (10 females and 11 males) and 40 pigtailed macaques (17 females and 23 males) to establish normal reference values for corneal nerve fibers that form the subbasal plexus. To determine whether age was associated with alterations in corneal subbasal nerve fibers (SNF), 
macaques were separated into four age groups (3y, 4 to $6 y, 7$ to 10y, and over 10 y); each age group was composed of 10 to 23 animals. Animals included in the study had no history or current clinical evidence of ocular disease, neurologic disease, or systemic diseases known to cause neurologic sequelae, such as diabetes mellitus.

For SIV studies,12 juvenile pigtailed macaques co-housed at JHU were inoculated intravenously with SIV/17E-Fr and SIVdeltaB670.(15) Animals had corneal confocal scans performed under ketamine sedation 10 to14 days before SIV inoculation and then at 10 days post-inoculation to determine whether corneal sensory nerve fiber alterations could be detected during acute SIV infection. All animal work was approved by the JHU IACUC and determined to be in accordance with the guidelines outlined in the Animal Welfare Act and Regulations (United States Department of Agriculture) and the $8^{\text {th }}$ edition of the Guide for the Care and Use of Laboratory Animals (National Institutes of Health).

Image acquisition. Central corneal images were acquired from all animals using the Heidelberg Retina Tomograph III (HRT III) Rostock Cornea Module (Heidelberg Engineering, Heidelberg, Germany). Each image covered a field of view of $400 \mu \mathrm{m}$ by $400 \mu \mathrm{m}$ over 384 by 384 pixels. Animals were anesthetized with 10mg/kg ketamine $\mathrm{HCl}$ (Zetamine, VetOne, Boise, ID) intramuscularly for imaging. If needed, animals received additional doses of either 5-10 mg/kg ketamine $\mathrm{HCl}$ intramuscularly or intravenously, 0.1-0.5mg/kg acepromazine (Acepromazine Maleate Injection, USP, VetOne, Boise, ID) intramuscularly, or 0.1-0.2mg/kg midazolam (Midazolam Injection, USP, Hospira, San Jose, CA) intramuscularly to extend anesthesia time. Exact drug and dosage regimens were determined by the veterinarian for 
each animal to maintain sedation for the duration of the imaging session. Once anesthetized, 1-2 drops of $0.5 \%$ proparacaine hydrochloride ophthalmic solution (Alcon, Fort Worth, TX) were applied topically to the right eye of each animal. A thin layer of a sterile lubricant eye gel (GenTeal Lubricant Eye Gel, Alcon, Fort Worth, TX) was also applied to the right eye of each animal and a speculum was placed to retract the eyelids throughout the imaging session. A thin layer of sterile lubricant eye gel was applied to the tip of the objective lens and a sterile disposable cap (Tomocap, Heidelberg Engineering, Heidelberg, Germany) was placed over the lens. The head of the anesthetized animal was manually positioned to orient the eye towards the corneal surface. Images of the subbasal nerve plexus of the cornea were acquired from each animal using a sequence scan. Imaging was performed until 5 highquality images of the subbasal nerve plexus (SBP) in the central cornea were obtained. Each imaging session lasted between 5 to 15 minutes.

Measuring Plasma SIV RNA. SIV RNA was measured by qRT-PCR using the QuantiTech Virus Kid (Qiagen) and the following primers and probes in the SIV gag region (Fwd: 5'GTCTGCGTCATCTGGTG CATTC-3', Rev: 5'-CACTAGGTGTCTCTGCAC TATCTGTTTTG- 3', Probe: 5'-CTTCCTCAGTGTGTTTCACTTTCTCTTCTG- 3') with the following cycling conditions $50^{\circ} \mathrm{C}$ for $30 \mathrm{~min}$ to reverse transcribe RNA, $94^{\circ} \mathrm{C}$ for $15 \mathrm{~min}$, which was followed by 45 cycles of PCR at $94^{\circ} \mathrm{C}$ for 15 seconds, $55^{\circ} \mathrm{C}$ for 15 seconds, and $60^{\circ} \mathrm{C}$ for 30 seconds as previously described.(5)

\section{Deep Learning-Based Analysis: Applying deepNerve for automated assessments}


As previously described in detail, we developed a customized deep learning-based approach termed deepNerve to measure objectively three parameters of interest in the macaque subbasal plexus: corneal nerve fiber length (CNFL), fractal dimension, and tortuosity. (16) This algorithm was specifically built using macaque corneal nerve images obtained by IVCM as current automated approaches designed to assess the SBP were developed for human studies. Image quality from macaque IVCM scans lags behind human image quality because 1) macaque eyes are smaller with a more acute radius of curvature and a thinner cornea leading to a lower signal to noise image in the periphery of the images, and 2) macaques need to be anesthetized and manually positioned for IVCM unlike compliant human subjects who can be directed to aid orientation of the cornea to the microscope objective. For each individual animal corneal scan, the 5 highest quality images of subbasal plexus were selected for deepNerve input. These highest quality corneal scan images from macaques were processed to obtain three distinct parameters:

1) Corneal nerve fiber length - the total lengths of the nerves divided by the area of the image $\left(\mathrm{mm} / \mathrm{mm}^{2}\right) \cdot(17)$

2) Fractal dimension - a measure of nerve complexity that assesses the nerve pattern over scale and records the spatial loss of nerves. We used the box counting method over the same scales as previously published. (18)

3) Tortuosity - the Euclidian distance between the end points of each nerve segment divided by the true length of that segment. The more tortuous the vessel is over its extent, the longer the traversable length relative to the Euclidian distance and the higher the metric. Nerve branch points are also included as end points defining a nerve segment. (16) 


\section{Data Analysis}

The median values for all parameters calculated by deepNerve for each individual corneal confocal scan were used as representative values for subsequent data analysis for each animal. Group comparisons between species and sex were performed using the t-test; age comparisons utilized ANOVA. The changes from baseline pre-infection to 10 days post-SIV infection within individual animals were analyzed using the paired t-test. All statistical analyses were performed using GraphPad Prism v. 8.0 with $\alpha=0.05$.

\section{Results}

While a recent report illustrated the salient architectural features of subbasal nerve fibers in macaques based on immunohistochemical staining of corneal whole mounts, corneal subbasal plexus nerve fiber morphology in IVCM images from macaques has not been reported previously.(19) In this study, visual comparison of corneal confocal scans obtained from a human subject (51 year old male), a pigtailed macaque (4 year old male), and a rhesus macaque (7 year old female) revealed similar nerve morphology in the subbasal plexus of the central cornea (Figure 1). All primate species exhibited comparable subbasal nerve plexus architecture with predominantly parallel arrangement of nerve fibers, and both branching of nerve fibers and interconnecting smaller diameter anastomoses between the main nerve fibers. Macaque nerves in general were observed to be thinner than human nerves. 


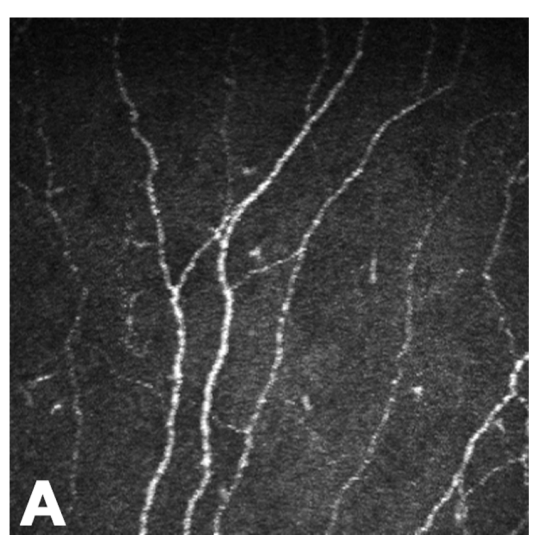

Human

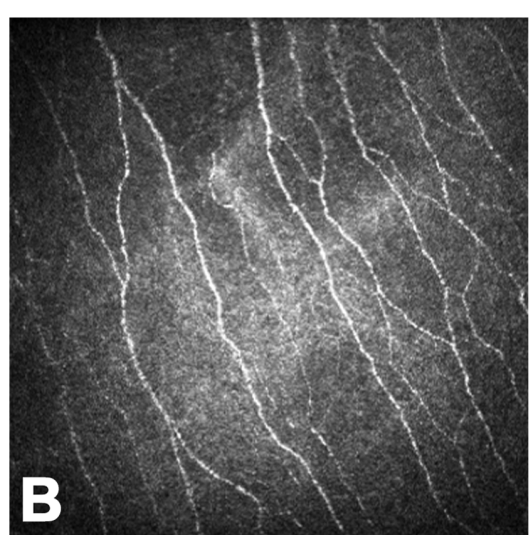

Pigtailed Macaque

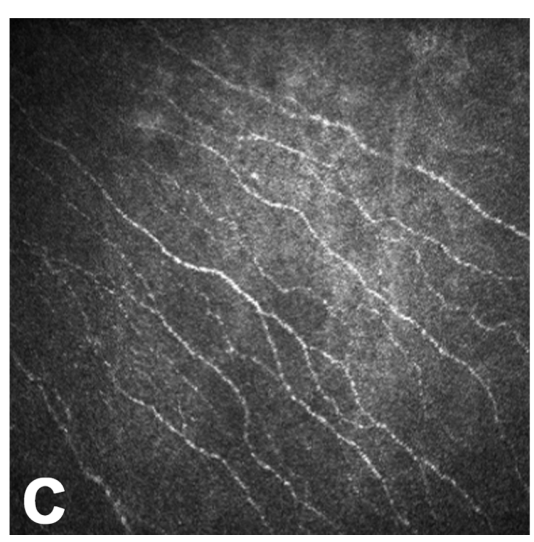

Rhesus Macaque

Figure 1. Representative images of the central corneal subbasal nerve plexus obtained by in vivo corneal confocal microscopy from A) a human, B) a pigtailed macaque, and C) a rhesus macaque illustrates similar density, arrangement, and morphology of the small sensory nerves across these primate species. Each image covers the same area of

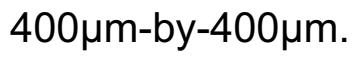

Corneal confocal scan images often have been assessed using manual tracing techniques that are inherently subjective and time-consuming. In contrast, nerve fiber detection by a customized deep learning-based approach rapidly and objectively identifies the large majority of individual nerve fibers in scans, facilitating subsequent automated measurements of nerve fiber parameters. This approach, termed deepNerve, was specifically designed to identify nerves present in macaque corneal scans as there is no current automated platform available for this purpose in macaques. (16)

After deepNerve processing, we compared corneal nerve parameters between healthy pigtailed macaques and rhesus macaques housed at JHU. There are notable differences between these species with regard to sensory nerve fiber innervation of the epidermis and 
SIV outcomes including extent of nervous system damage, thus establishing and comparing normative data is extremely valuable to enhance the rigor and reproducibility of macaquebased research.(20) There were no significant differences identified between normal pigtailed macaques versus comparable rhesus macaques for corneal nerve fiber length or fractal dimension; however, nerve fiber tortuosity was significantly higher in pigtailed macaques than rhesus macaques $(P=0.005 ;$ Fig 2; Table 1).
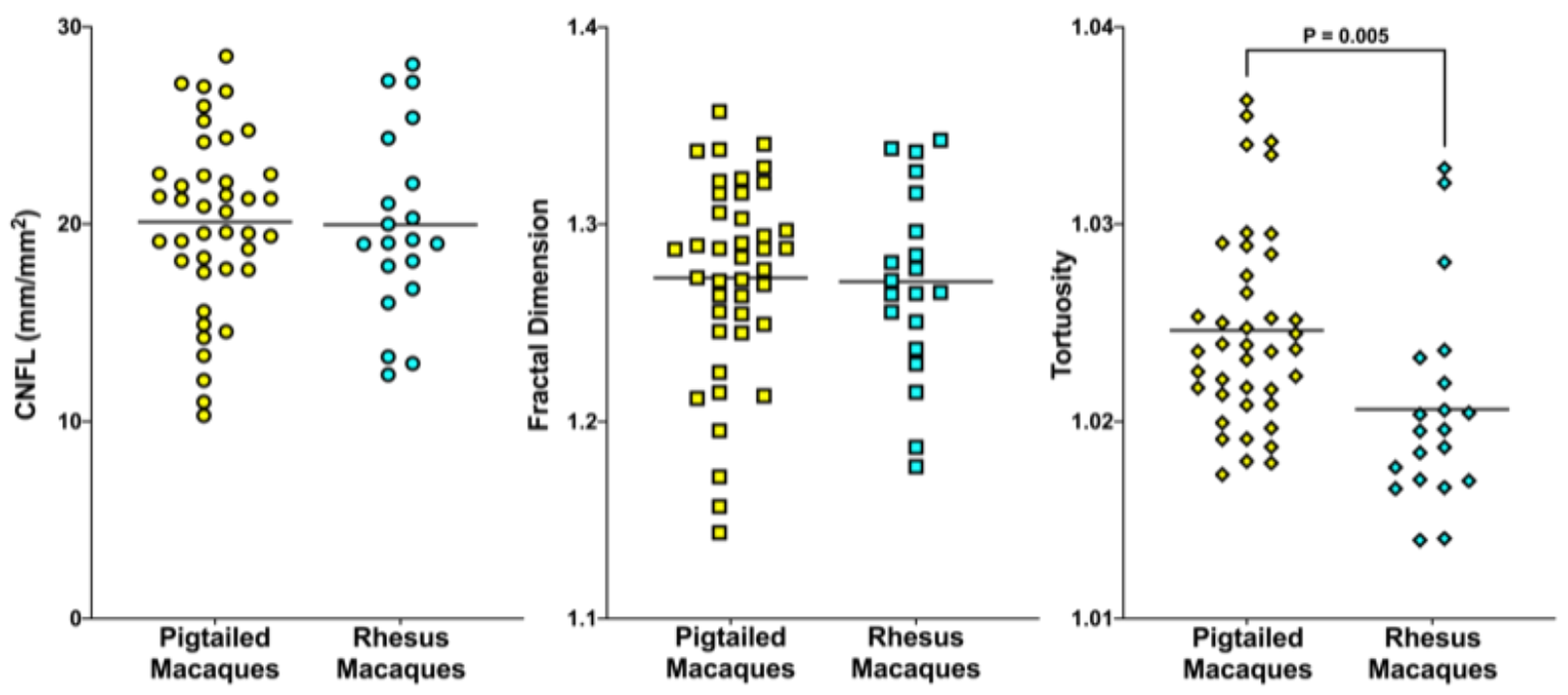

Figure 2. CNFL (left) and Fractal Dimension (center) did not differ significantly between pigtailed macaques versus rhesus macaques. However, a significantly higher tortuosity was observed in pigtailed macaques compared with rhesus macaques (right).

As sex can be a major variable for many biological measurements, comparisons of corneal nerve parameters were made between female macaques versus male macaques for all corneal measurements. No significant differences were identified in these comparisons, 
suggesting that baseline corneal nerve parameters do not differ between males and females (Figure 3, Table 1), similar to reports in humans.(21)

The influence of age on corneal nerve parameters was examined after categorizing healthy animals into one of four age groups, (Figure 4, Table 1). Although there was a trend for tortuosity to increase with age in animals older than 10 years, this was not a significant finding. As we had fewer animals in the older age group, it is possible that a larger scale study might find a significant increase with maturation. Animals past breeding age were not included in this study as it was based in the JHU breeding colony; follow up studies in aging macaque cohorts may reveal age-related alterations in corneal sensory innervation. Of note, reduced corneal nerve fiber density has been reported with aging in both mice and humans of advanced age. $(21,22)$
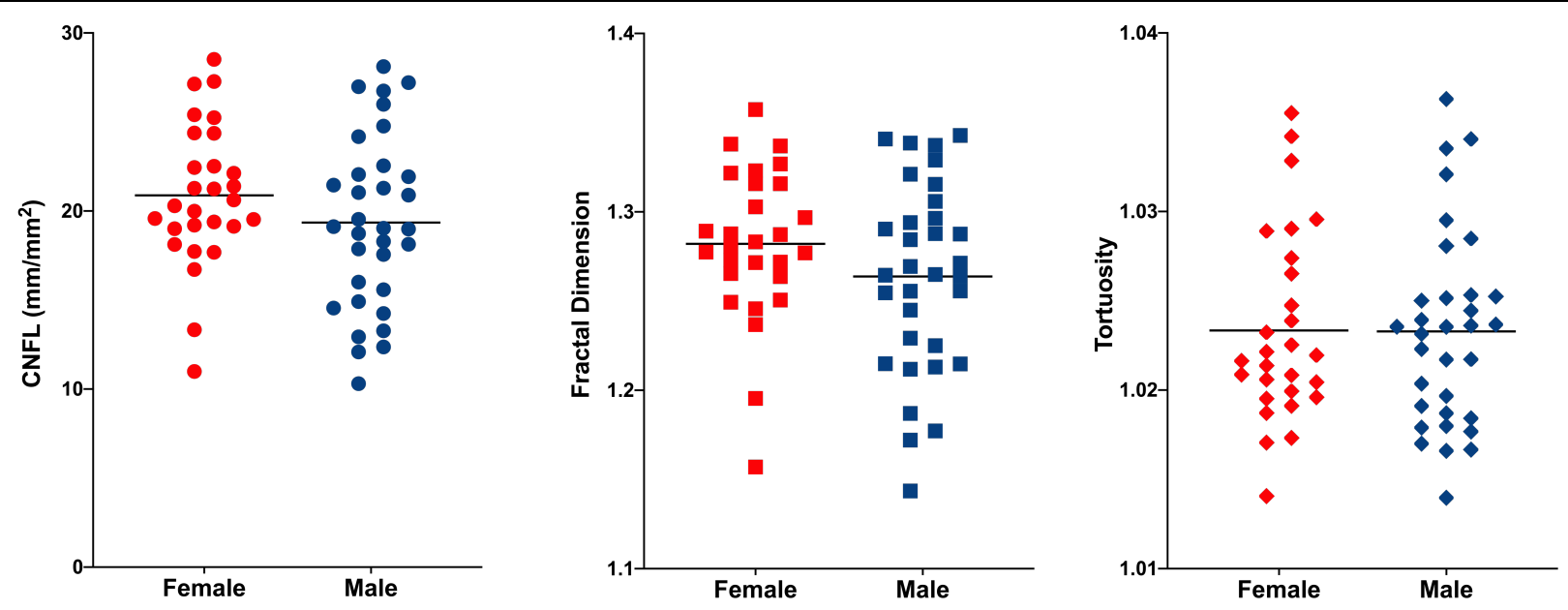

Figure 3. CNFL (left), Fractal Dimension (center), and Tortuosity (right) did not differ significantly between male versus female macaques. 
bioRxiv preprint doi: https://doi.org/10.1101/2020.04.19.048926; this version posted April 20, 2020. The copyright holder for this preprint (which was not certified by peer review) is the author/funder, who has granted bioRxiv a license to display the preprint in perpetuity. It is made available under aCC-BY-NC-ND 4.0 International license.

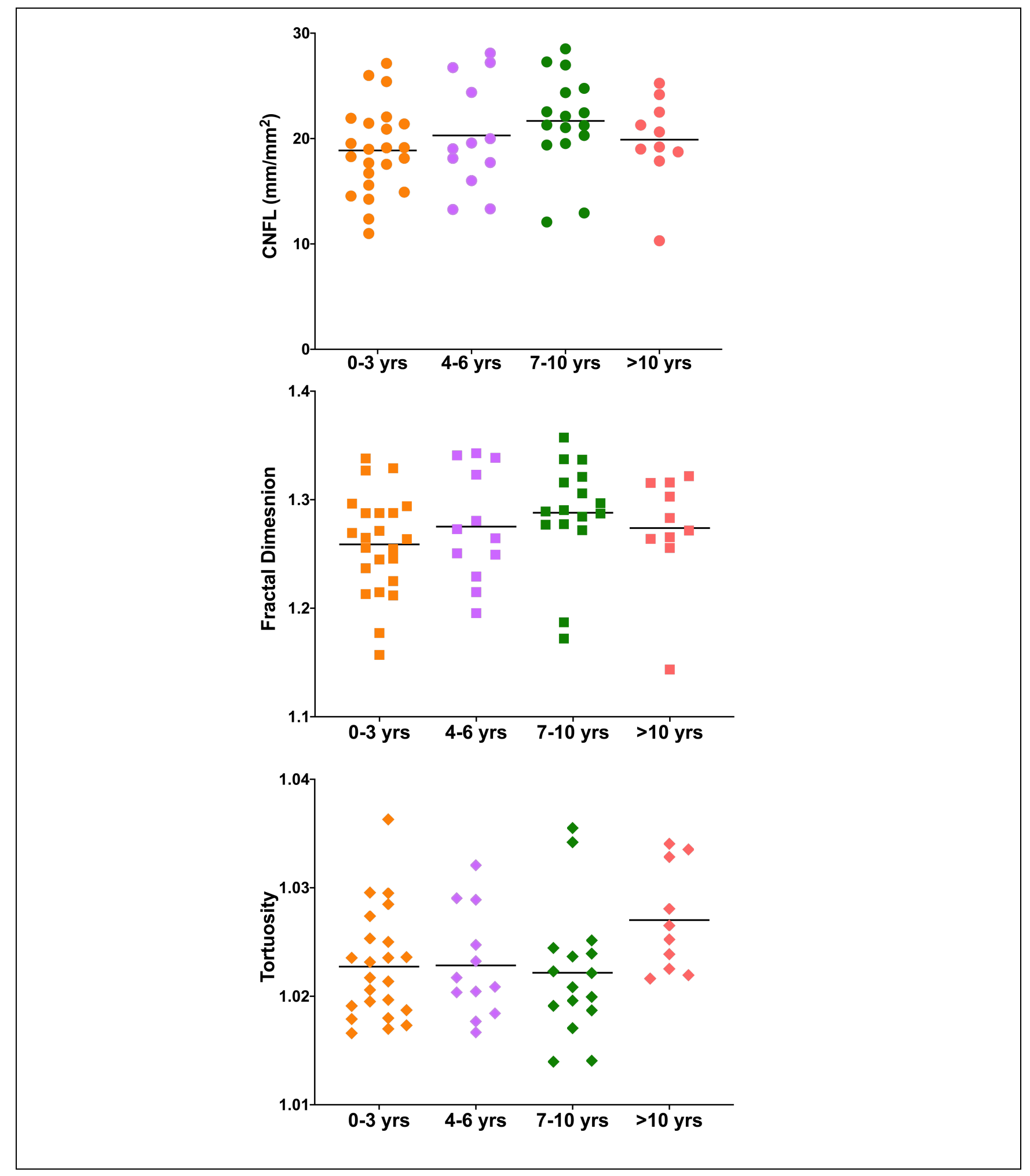


Figure 4. CNFL (top), Fractal Dimension (center), and Tortuosity (bottom) stratified by age.

Only tortuosity was higher in the oldest group, but not significantly.

Table 1. Normative values for macaque IVCM corneal nerve parameters

\begin{tabular}{|c|c|c|c|c|c|c|}
\hline \multirow[b]{2}{*}{ Species } & \multicolumn{2}{|c|}{ CNFL $\left(\mathrm{mm} / \mathrm{mm}^{2}\right)$} & \multicolumn{2}{|c|}{ Fractal Dimension } & \multicolumn{2}{|c|}{ Tortuosity } \\
\hline & MEAN & SD & MEAN & SD & MEAN & SD \\
\hline Pigtailed & 20.10 & 4.46 & 1.27 & 0.05 & 1.025 & 0.005 \\
\hline Rhesus & 19.96 & 4.68 & 1.27 & 0.05 & 1.021 & 0.005 \\
\hline Sex & & & & & & \\
\hline Female & 20.88 & 3.97 & 1.28 & 0.04 & 1.023 & 0.005 \\
\hline Male & 19.35 & 4.84 & 1.26 & 0.05 & 1.023 & 0.005 \\
\hline Age & & & & & & \\
\hline $0-3$ yrs & 18.87 & 4.15 & 1.26 & 0.05 & 1.022 & 0.004 \\
\hline $4-6$ yrs & 20.29 & 5.20 & 1.28 & 0.01 & 1.023 & 0.005 \\
\hline $7-10$ yrs & 21.68 & 4.51 & 1.29 & 0.01 & 1.022 & 0.006 \\
\hline$>10$ yrs & 19.89 & 4.15 & 1.27 & 0.02 & 1.027 & 0.005 \\
\hline
\end{tabular}




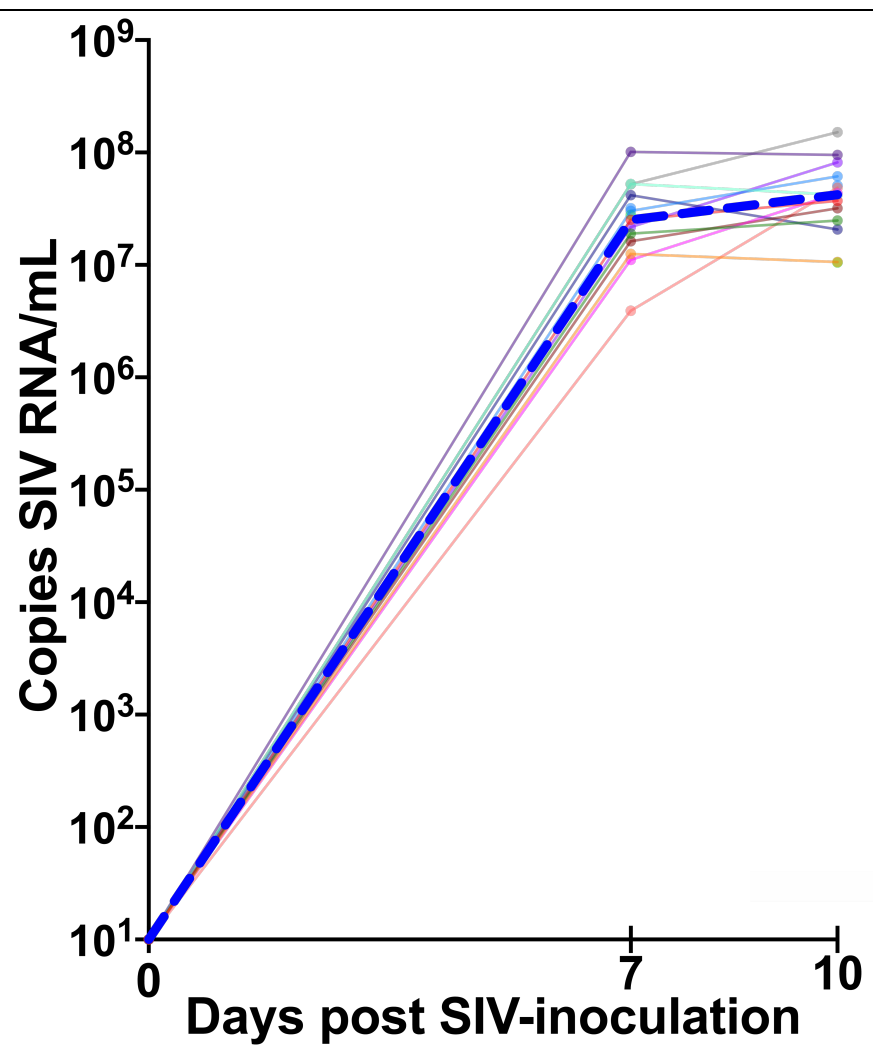

Figure 5. All 12 SIV-infected macaques had comparable high plasma viral loads during the acute phase of SIV infection with the peak group median plasma viral load (blue line) of 4.2 $\times 10^{7}$ copies $/ \mathrm{mL}$ at day 10 post-inoculation. Individual animal plasma viral loads are represented by the thin colored lines. 


\section{Raw Scan Image}
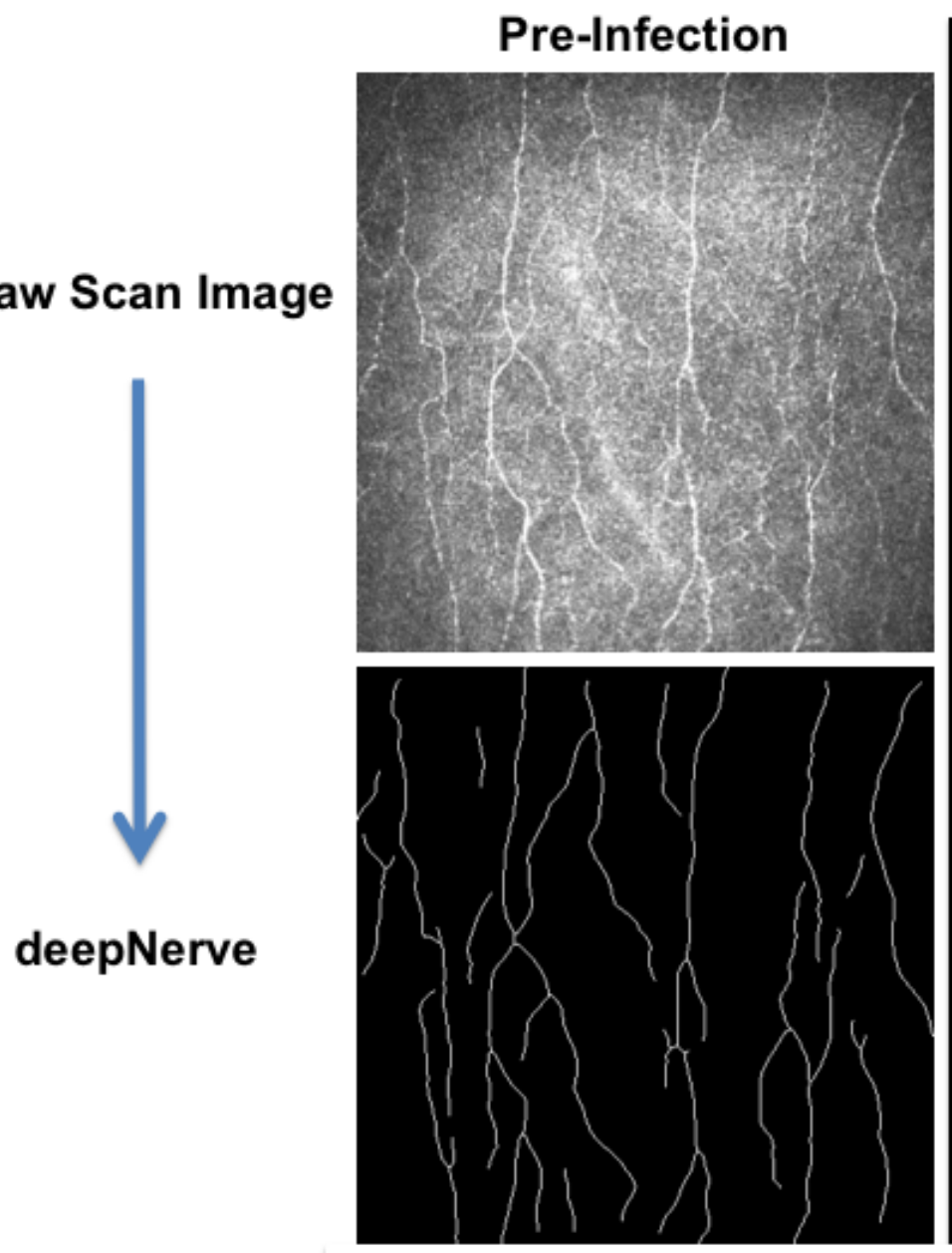

10 Days post-SIV
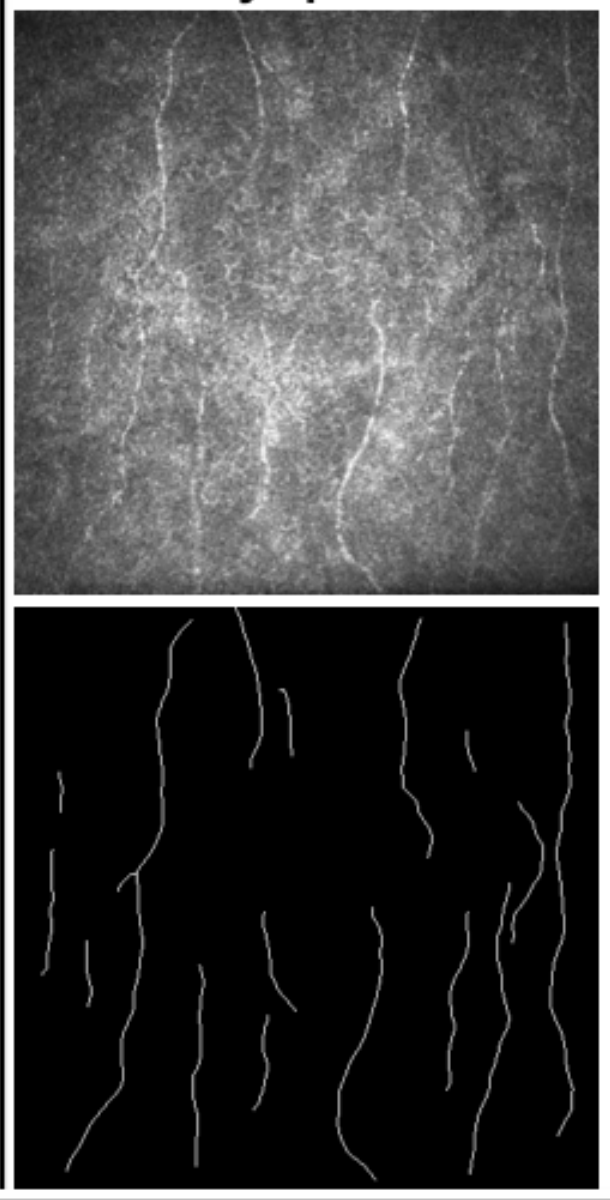

Fig 6. IVCM images (top) and deepNerve representations of the same images of corneal nerve fibers (below) from a pigtailed macaque at pre-infection (left column) and 10 days post-SIV infection (right column) illustrate loss of subbasal nerve fibers with SIV. 
Our previous studies of HIV-induced damage to the peripheral nervous system based in the SIV/pigtailed macaque model have revealed significant loss of small sensory nerve fibers in the epidermis of the skin in the SIV/pigtailed macaque model at 14 days post-SIV infection (25); similar early loss of epidermal nerve fibers was found in a rhesus macaque SIV model 21 days post-SIV infection.(23) In this study, to determine whether IVCM-based analysis could detect early sensory nerve fiber damage, we performed IVCM on 12 pigtailed macaques to obtain pre-infection baseline corneal nerve data followed by IVCM scans on day 10 days after infection with SIV. All infected animals had similar plasma viral loads (Fig 5). A decline in CNFL density was visually apparent when pre-infection IVCM scans and deepNerve processed images were compared (Fig 6).
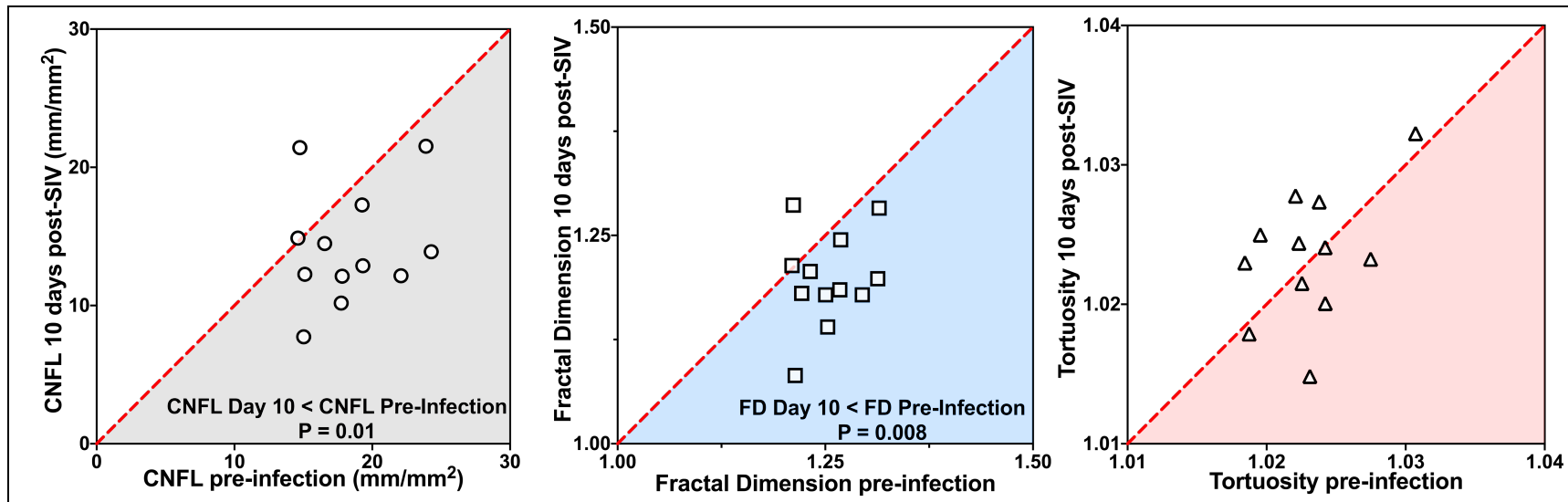

Figure 7. 10 of the 12 SIV-infected macaques had lower corneal nerve fiber counts 10 days post-inoculation with SIV than their baseline pre-infection corneal scan values (left panel; P $=0.01$, paired t-test). Similarly, fractal dimension declined with SIV infection in of 12 animals (middle panel; $\mathrm{P}=0.008$, paired t-test). In contrast, tortuosity was not altered during acute infection (right panel). 
deepNerve- based measurements of subbasal nerve fiber features demonstrated that CNFL declined significantly by day 10 of infection $(P=0.01$, paired t-test, Figure 7) with 10 of the 12 macaques having lower measurements at day 10 consistent with sensory nerve fiber loss. In contrast with the SIV-infected macaques, corneal nerve fiber length in uninfected control animals imaged at comparable repeat timepoints were not significantly different $(P=0.34$; paired t-test). These data demonstrate the ability of the combination of IVCM and automated analysis to detect corneal nerve fiber loss during acute infection, an early timepoint when SIV replicates in sensory ganglia macrophages and induces both immune activation and metabolic alterations. $(4,7,24)$

Similar to corneal nerve fiber length, fractal dimension also declined with SIV infection with 10 of the 12 animals with lower counts after SIV infection (Figure 7; $P=0.008$, paired t-test). In contrast, corneal nerve fiber tortuosity was not significantly altered during acute infection and this may not be a sensitive marker of relatively acute damage.

\section{Discussion}

Asian macaques are frequently studied to understand a variety of diseases including those that involve loss of small sensory nerve fibers associated with development of peripheral neuropathy.(19) Studies of both pigtailed macaques (Macaca nemestrina) and rhesus macaques (Macaca mulatta), for example, have been highly informative for understanding HIV pathogenesis including peripheral nerve damage that underlies development of HIV sensory neuropathy.(4-7, 24-26) The current clinical standard for measuring sensory nerve 
fiber loss in human and macaque subjects is performing counts of epidermal nerve fibers present in skin biopsies.(27) This technique is invasive and requires extensive tissue processing and analysis making repeat sampling for longitudinal assessments challenging. In contrast, non-invasive in vivo corneal confocal microscopy can quickly collect multiple images of the small sensory nerve fibers comprising the corneal subbasal plexus in a non-invasive manner, facilitating longitudinal acquisition of data. These images then can be rapidly evaluated using advanced automated processing approaches that objectively measure alterations in morphologic features.(28-33)

We have previously demonstrated corneal sensory nerve fiber loss in the SIV/pigtailed macaque model of HIV-induced PNS damage by studying corneal innervation ex vivo using immunohistochemical staining for the nerve marker $\beta$ III-tubulin followed by automated image analysis.(14) Corneal nerve fiber loss was well correlated with decline in epidermal nerve fibers. This study built on those findings by using IVCM to collect images of the macaque subbasal plexus, adapting techniques established in human studies.(12)

Using our well-characterized SIV/pigtailed macaque model of HIV-induced PNS damage, to determine whether acute SIV infection altered sensory nerves in the subbasal plexus, we obtained repeat IVCM images of 12 animals both pre-infection and then at 10 days postinfection time-points allowing us to directly compare corneal nerve fiber alterations in individual subjects. Automated analysis of IVCM images revealed that 10 of the 12 animals developed significant corneal nerve fiber alterations consistent with sensory nerve fiber damage and loss. Our earlier studies have shown that SIV replicates in macrophages in the 
trigeminal ganglia as early as 7 days post-infection.(4) Furthermore, we have used targeted transcriptomic analyses to reveal that immune activation and metabolic alterations can be detected in dorsal root ganglia 7 days post-SIV infection.(24) These changes in sensory ganglia during acute SIV infection likely underlie the sensory nerve fiber loss detected by both IVCM and skin biopsy-based measurements of epidermal nerve fiber density. Understanding acute alterations induced by HIV by studies in the SIV/macaque model is important because effective neuroprotective interventions are most valuable at the earliest stages of nerve damage.

A recent cross-sectional study showed that IVCM can detect corneal nerve fiber loss in people living with HIV.(13) A separate study of HIV cohorts demonstrated signs of neuropathy in $35 \%$ of individuals during the first months primary HIV infection before starting ART, suggesting that HIV causes early damage to the sensory nervous system as reported in SIV.(3) Future studies tracking corneal nerve fiber alterations in people living with HIV before and while on ART will contribute to our understanding of the pathogenesis of HIV neuropathy and enhance efforts to prevent and treat this debilitating disease.

To address the more challenging imaging conditions resulting from the aforementioned difference in macaque anatomy and lack of compliance with respect to human subjects, we developed a customized deep learning-based approach to assess macaque corneal nerve parameters in support of macaque IVCM studies. (16) We used this combination of IVCM and automated analysis to generate normative reference data sets for female and male pigtailed and rhesus macaques of varying ages. We did not detect significant species, sex, or 
age differences in measured corneal nerve parameters, however the approximately two-fold range in variation found in normal animals illustrates the challenge of performing crosssectional studies if group sizes of animals are relatively small.

The ability to track corneal nerve fiber alterations longitudinally in individual animals by IVCM will enhance the rigor and reproducibility of macaque-based studies that are typically limited in size given animal availability constraints and high cost of macaque studies. Future studies will explore the potential of our deep learning-based approach for analyzing corneal nerve fiber properties in human subjects including those living with HIV to help guide prevention and clinical management of neuropathy. 


\section{References}

1. Ellis RJ, Rosario D, Clifford DB, McArthur JC, Simpson D, Alexander T, et al. Continued high prevalence and adverse clinical impact of human immunodeficiency virusassociated sensory neuropathy in the era of combination antiretroviral therapy: the CHARTER Study. Arch Neurol. 2010;67(5):552-8.

2. McArthur JC, Brew BJ, Nath A. Neurological complications of HIV infection. Lancet Neurol. 2005;4(9):543-55.

3. Wang SX, Ho EL, Grill M, Lee E, Peterson J, Robertson K, et al. Peripheral neuropathy in primary HIV infection associates with systemic and central nervous system immune activation. J Acquir Immune Defic Syndr. 2014;66(3):303-10.

4. Laast VA, Pardo CA, Tarwater PM, Queen SE, Reinhart TA, Ghosh M, et al. Pathogenesis of simian immunodeficiency virus-induced alterations in macaque trigeminal ganglia. J Neuropathol Exp Neurol. 2007;66(1):26-34.

5. Laast VA, Shim B, Johanek LM, Dorsey JL, Hauer PE, Tarwater PM, et al. Macrophage-mediated dorsal root ganglion damage precedes altered nerve conduction in SIV-infected macaques. Am J Pathol. 2011;179(5):2337-45.

6. Mangus LM, Dorsey JL, Laast VA, Ringkamp M, Ebenezer GJ, Hauer P, et al. Unraveling the pathogenesis of HIV peripheral neuropathy: insights from a simian immunodeficiency virus macaque model. ILAR J. 2014;54(3):296-303.

7. Ebenezer GJ, McArthur JC, Polydefkis M, Dorsey JL, O'Donnell R, Hauer P, et al. SIVinduced impairment of neurovascular repair: a potential role for VEGF. J Neurovirol. 2012;18(3):222-30. 
8. Polydefkis M, Yiannoutsos CT, Cohen BA, Hollander H, Schifitto G, Clifford DB, et al. Reduced intraepidermal nerve fiber density in HIV-associated sensory neuropathy. Neurology. 2002;58(1):115-9.

9. Malik RA, Kallinikos P, Abbott CA, van Schie CH, Morgan P, Efron N, et al. Corneal confocal microscopy: a non-invasive surrogate of nerve fibre damage and repair in diabetic patients. Diabetologia. 2003;46(5):683-8.

10. Tavakoli M, Quattrini C, Abbott C, Kallinikos P, Marshall A, Finnigan J, et al. Corneal confocal microscopy: a novel noninvasive test to diagnose and stratify the severity of human diabetic neuropathy. Diabetes Care. 2010;33(8):1792-7.

11. Wang EF, Misra SL, Patel DV. In Vivo Confocal Microscopy of the Human Cornea in the Assessment of Peripheral Neuropathy and Systemic Diseases. Biomed Res Int. $2015 ; 2015: 951081$.

12. Patel DV, McGhee CN. Mapping of the normal human corneal sub-Basal nerve plexus by in vivo laser scanning confocal microscopy. Invest Ophthalmol Vis Sci. 2005;46(12):44858.

13. Kemp HI, Petropoulos IN, Rice ASC, Vollert J, Maier C, Sturm D, et al. Use of Corneal Confocal Microscopy to Evaluate Small Nerve Fibers in Patients With Human Immunodeficiency Virus. JAMA Ophthalmol. 2017;135(7):795-800.

14. Dorsey JL, Mangus LM, Oakley JD, Beck SE, Kelly KM, Queen SE, et al. Loss of corneal sensory nerve fibers in SIV-infected macaques: an alternate approach to investigate HIV-induced PNS damage. Am J Pathol. 2014;184(6):1652-9. 
15. Beck SE, Queen SE, Metcalf Pate KA, Mangus LM, Abreu CM, Gama L, et al. An

SIV/macaque model targeted to study HIV-associated neurocognitive disorders. J Neurovirol. 2018;24(2):204-12.

16. Oakley JD, Russakoff DB, McCarron ME, Weinberg RL, Izzi JM, Mankowski JL. Deep Learning-Based Analysis of Macaque Corneal Sub-Basal Nerve Fibers in Confocal Microscopy Images. bioRxiv. 2019:758433.

17. Dabbah MA, Graham J, Petropoulos IN, Tavakoli M, Malik RA. Automatic analysis of diabetic peripheral neuropathy using multi-scale quantitative morphology of nerve fibres in corneal confocal microscopy imaging. Med Image Anal. 2011;15(5):738-47.

18. Chen X, Graham J, Petropoulos IN, Ponirakis G, Asghar O, Alam U, et al. Corneal Nerve Fractal Dimension: A Novel Corneal Nerve Metric for the Diagnosis of Diabetic Sensorimotor Polyneuropathy. Invest Ophthalmol Vis Sci. 2018;59(2):1113-8.

19. Marfurt C, Anokwute MC, Fetcko K, Mahony-Perez E, Farooq H, Ross E, et al. Comparative Anatomy of the Mammalian Corneal Subbasal Nerve Plexus. Invest Ophthalmol Vis Sci. 2019;60(15):4972-84.

20. Mangus LM, Dorsey JL, Weinberg RL, Ebenezer GJ, Hauer P, Laast VA, et al. Tracking Epidermal Nerve Fiber Changes in Asian Macaques: Tools and Techniques for Quantitative Assessment. Toxicol Pathol. 2016;44(6):904-12.

21. Niederer RL, Perumal D, Sherwin T, McGhee CN. Age-related differences in the normal human cornea: a laser scanning in vivo confocal microscopy study. Br J Ophthalmol. 2007;91(9):1165-9.

22. De Silva MEH, Hill LJ, Downie LE, Chinnery HR. The Effects of Aging on Corneal and Ocular Surface Homeostasis in Mice. Invest Ophthalmol Vis Sci. 2019;60(7):2705-15. 
23. Lakritz JR, Robinson JA, Polydefkis MJ, Miller AD, Burdo TH. Loss of intraepidermal nerve fiber density during SIV peripheral neuropathy is mediated by monocyte activation and elevated monocyte chemotactic proteins. J Neuroinflammation. 2015;12:237.

24. Mangus LM, Weinberg RL, Knight AC, Queen SE, Adams RJ, Mankowski JL. SIVInduced Immune Activation and Metabolic Alterations in the Dorsal Root Ganglia During Acute Infection. J Neuropathol Exp Neurol. 2019;78(1):78-87.

25. Dorsey JL, Mangus LM, Hauer P, Ebenezer GJ, Queen SE, Laast VA, et al. Persistent Peripheral Nervous System Damage in Simian Immunodeficiency Virus-Infected Macaques Receiving Antiretroviral Therapy. J Neuropathol Exp Neurol. 2015;74(11):1053-60.

26. Lakritz JR, Bodair A, Shah N, O'Donnell R, Polydefkis MJ, Miller AD, et al. Monocyte Traffic, Dorsal Root Ganglion Histopathology, and Loss of Intraepidermal Nerve Fiber Density in SIV Peripheral Neuropathy. Am J Pathol. 2015;185(7):1912-23.

27. Mangus LM, Rao DB, Ebenezer GJ. Intraepidermal Nerve Fiber Analysis in Human Patients and Animal Models of Peripheral Neuropathy: A Comparative Review. Toxicol Pathol. 2019:192623319855969.

28. Al-Fahdawi S, Qahwaji R, Al-Waisy AS, Ipson S, Malik RA, Brahma A, et al. A fully automatic nerve segmentation and morphometric parameter quantification system for early diagnosis of diabetic neuropathy in corneal images. Comput Methods Programs Biomed. 2016;135:151-66.

29. Annunziata R, Kheirkhah A, Aggarwal S, Hamrah P, Trucco E. A fully automated tortuosity quantification system with application to corneal nerve fibres in confocal microscopy images. Medical image analysis. 2016;32:216-32. 
30. Chen X, Graham J, Dabbah MA, Petropoulos IN, Tavakoli M, Malik RA. An Automatic Tool for Quantification of Nerve Fibers in Corneal Confocal Microscopy Images. IEEE Trans Biomed Eng. 2017;64(4):786-94.

31. Guimarães P, Wigdahl J, Ruggeri A. A Fast and Efficient Technique for the Automatic Tracing of Corneal Nerves in Confocal Microscopy. Translational Vision Science \& Technology. 2016;5(5).

32. Scarpa F, Grisan E, Ruggeri A. Automatic recognition of corneal nerve structures in images from confocal microscopy. Investigative ophthalmology \& visual science. 2008;49(11):4801-7. 